\title{
Minimal manipulability: anonymity and surjectivity
}

Citation for published version (APA):

Maus, S., Peters, H. J. M., \& Storcken, A. J. A. (2004). Minimal manipulability: anonymity and surjectivity. METEOR, Maastricht University School of Business and Economics. METEOR Research Memorandum No. 007 https://doi.org/10.26481/umamet.2004007

Document status and date:

Published: 01/01/2004

DOI:

10.26481/umamet.2004007

Document Version:

Publisher's PDF, also known as Version of record

\section{Please check the document version of this publication:}

- A submitted manuscript is the version of the article upon submission and before peer-review. There can be important differences between the submitted version and the official published version of record.

People interested in the research are advised to contact the author for the final version of the publication, or visit the DOI to the publisher's website.

- The final author version and the galley proof are versions of the publication after peer review.

- The final published version features the final layout of the paper including the volume, issue and page numbers.

Link to publication

\footnotetext{
General rights rights.

- You may freely distribute the URL identifying the publication in the public portal. please follow below link for the End User Agreement:

www.umlib.nl/taverne-license

Take down policy

If you believe that this document breaches copyright please contact us at:

repository@maastrichtuniversity.nl

providing details and we will investigate your claim.
}

Copyright and moral rights for the publications made accessible in the public portal are retained by the authors and/or other copyright owners and it is a condition of accessing publications that users recognise and abide by the legal requirements associated with these

- Users may download and print one copy of any publication from the public portal for the purpose of private study or research.

- You may not further distribute the material or use it for any profit-making activity or commercial gain

If the publication is distributed under the terms of Article $25 \mathrm{fa}$ of the Dutch Copyright Act, indicated by the "Taverne" license above, 


\title{
Minimal Manipulability: Anonymity and Sur- jectivity
}

\author{
Stefan Maus, Hans Peters, Ton Storcken \\ Date of this version: March 19, 2004 \\ University of Maastricht, Department of Quantitative Economics, \\ P.O. Box 616, 6200 MD Maastricht, THE NETHERLANDS \\ Tel.: +31433883761; fax: +31433884874 \\ Email: s.maus@ke.unimaas.nl, h.peters@ke.unimaas.nl, \\ t.storcken@ke.unimaas.nl
}

\begin{abstract}
Gibbard's (1973) and Satterthwaite's (1975) result implies that anonymous surjective social choice functions on more than two alternatives are manipulable. Placing some mild constraints on the number of agents compared to the number of alternatives, we show what the minimal number of manipulable profiles of such social choice functions is. Moreover, all such social choice functions attaining the lower bound are characterized. They show a trade off between minimizing manipulability and treating alternatives neutrally.
\end{abstract}

\section{Introduction}

A well-known result of Gibbard (1973) and Satterthwaite (1975) states that any strategy-proof surjective social choice function on more than two alternatives is dictatorial. This implies that anonymity and surjectivity are not compatible with strategy-proofness. Here we investigate how incompatible these are, i..e. how much manipulability we have to allow at anonymous and surjective social choice functions.

There are a few studies on the degree of vulnerability for strategic behaviour of classical social choice functions, such as Borda, Plurality, etc.. For example, Aleskerov (1999) contains simulation and enumeration results on 26 different social choice functions for different indices of manipulability. Slinko (2002) counts the number of instabile profiles of classical social choice functions, which is an upper bound for the number of manipulable profiles of these social choice functions. On the other hand, little is known about the 
extent of manipulability that social choice functions satisfying certain properties must admit. Results on this may provide lower bounds to which then all social choice functions satisfying these properties can be compared. An investigation was pioneered by Kelly (1988), who gave the minimal number of manipulable profiles for nondictatorial surjective social choice functions with three alternatives and two agents and formulated several conjectures. This line of research was continued by Fristrup and Keiding (1998), who gave the minimal number of manipulable profiles for two agents and any number of alternatives. Maus et al. (2004) consider the three alternative case and show that for any number of agents larger than two there are six minimally manipulable nondictatorial surjective social choice functions, and these are even anonymous. However, they also consider minimally manipulable unanimous and nondictatorial social choice functions. These turn out to be nondictatorial only in a minimal sense, namely at only one profile.

Here we will exclude such social choice functions by demanding that agents are treated anonymously and we replace unanimity by surjectivity. In particular, anonymous social choice functions are nondictatorial. So, we are interested in the minimal number of manipulable profiles that an anonymous surjective social choice function has to admit. It is well known that in case of two alternatives any monotonic social choice function is strategy-proof. As monotonicity and anonymity are compatible (see e.g. May (1952)) in case of two alternatives this minimal number is zero. In case of three alternatives the minimal number is equal to the number of agents. This follows from Maus et al. (2004). Actually, if there are more than two agents the only social choice functions reaching this minimal number are anonymous. To the best of our knowledge only so far the problem to find the minimal number of manipulable profiles of anonymous surjective social choice functions is completely solved. Although here for four or more alternatives this minimal number is determined for many cases, there are still some cases which are completely open. Due to the combinatorics used in the proofs only the following cases are solved: The four alternatives and at least five agents case and the five or more alternatives and at least two more agents than alternatives case. So, roughly speaking, the problem is not solved if there are at least as many alternatives as agents. Up till now we could neither solve the problem for any of these cases by a different proof nor could we find counterexamples. Therefore the problem is completely open for these cases.

The characterization we provide is unified for all numbers of alternatives. Interpreting the definition with two alternatives boils down to a strategyproof social choice function for two alternatives called status-quo voting. In the three alternatives case it defines the minimally manipulable nondicatorial surjective social choice functions, as found in Maus et al. (2004). 
In general the characterized social choice functions show how one can (but probably would not want to) minimize manipulability while treating agents anonymously. They minimize also surjectivity, meaning that except for two alternatives, say $b$ and $c$, for all other alternatives there is precisely one profile at which they are chosen. At the remaining profiles the choice between $b$ and $c$ is on a unanimity basis: $c$ is chosen unless $b$ is unanimously preferred to $b$. So, alternatives are treated in a nonneutral way, which will usually be just as undesirable as treating agents unequal. Nevertheless the result shows what is possible if we want to minimize manipulability.

It should be noted that the way in which we measure manipulability, namely by counting manipulable profiles, is not the only possible one. In fact there are a lot of variations of this, where for example profiles are counted by the number of agents that can manipulate them, or the severity of the manipulation measured by the distance between what the agent achieves with and without manipulation is taken into account. Aleskerov (1999) contains an overview of such variations and numerical results on the manipulability of 26 social choice functions according to these measurements.

Within the restrictions pointed out we show as a main step of our proof of the main result that strategy-proof and anonymous surjective social choice between more than two alternatives is not possible on subsets of the whole domain satisfying a certain diversity condition, namely on subsets where agents hold at least $k \in \mathbb{N}$ different preferences. We point this out here since this is an impossibility result on a restricted domain that is of interest on its own. It says, that the impossibility result of Gibbard-Satterthwaite cannot be resolved by demanding diversity of preferences. The reader who is familiar with a proof of Arrow's theorem (Arrow 1963) and a proof GibbardSatterthwaite's theorem building on Arrow's theorem will recognize a lot of similarities. The basic idea is to show that the steps taken there can be adopted to go through also on the restricted domains considered here. To ensure that these steps can be adopted we need the restrictions that then cause the restrictions on the main result.

In the next section the relevant definitions are given, and the relation to Gibbard (1973), Satterthwaite (1975) and Maus et al. (2004) is made more precise. Sections 4 and 5 link strategy-proofness to monotonicity, Pareto optimality and decisiveness on sets of profiles where agents hold at least $k$ different preferences. Then, Section 6 combines this to show that there are no strategy-proof and anonymous surjective social choice functions selecting among more than two alternatives on these restricted domains. After that, Section 7 characterizes the minimally manipulable anonymous surjective social choice functions selecting from more than three alternatives. Finally, Section 8 concludes. 


\section{Preliminaries}

We denote the cardinality of a set $S$ by $|S|$.

Let $A$ be a finite set of alternatives, $m:=|A| \geq 3$, and $N$ a finite set of agents, $n:=|N| \geq 2$.

Let $t \subset A \times A$. We call $t$ complete if for all $x, y \in A(x, y) \in t$ or $(y, x) \in t$. Note that completeness of $t$ implies $(x, x) \in t$ for all $x \in A$. We call $t$ transitive if for all $x, y, z \in A(x, y) \in t$ and $(y, z) \in t$ implies $(x, z) \in A$. We call $t$ antisymmetric if for all $x, y \in A(x, y) \in t$ and $(y, x) \in t$ implies that $x=y$.

A preference $t \subset A \times A$ is a linear ordering (complete, transitive, antisymmetric) on $A$. Let $P$ denote the set of all preferences. Suppose that $A=\left\{x_{1}, x_{2}, \ldots x_{m}\right\}$. By completeness, transitivity and antisymmetry we can write conveniently

$$
t=x_{1} x_{2} \ldots x_{m}
$$

for the preference $t$ such that $\left(x_{i}, x_{j}\right) \in t$ if and only if $i \geq j, i, j \in$ $\{1,2, \ldots, m\}$, and

$$
t=\ldots x \ldots y \ldots
$$

if we want to express only that $x$ is strictly preferred to $y$.

A profile $p$ is a map from $N$ to $P$. Let $P^{N}$ denote the set of all these maps. Thus, a profile assigns to every agent $i$ a preference $p(i)$ over the alternatives. For a nonempty subset $S$ of $N$ we denote by $\left.p\right|_{S}$ the restriction of the map $p$ to the domain $S$. We denote a profile such that all agents have the same preference $t \in P$ by $t^{N}$.

A social choice function is a function $f: P^{N} \rightarrow A$. Hence, a social choice function selects a unique alternative $f(p)$ at every profile $p$.

A social choice function is called surjective if any alternative in $A$ is chosen at least once, i.e. if $f\left(P^{N}\right)=A$. In the literature this is also known as citizen-sovereignity. Throughout this paper we assume that any social choice function is surjective, and do not mention this anymore explicitly.

For a permutation $\sigma$ of $N$ and a profile $p \in P^{N}$ let $p \circ \sigma$ be the profile given by $(p \circ \sigma)(i):=p(\sigma(i))$ for all $i \in N$. A social choice function is called anonymous if $f(p)=f(p \circ \sigma)$ for all permutations $\sigma$ of $N$. Thus, anonymous social choice functions are symmetric in the arguments. In a sense they treat agents equally.

In contrast to anonymity, the following dictatorial social choice functions dict $_{d}$ respect only the preference of one single agent $d \in N$, the dictator. For any profile $p$ dict $_{d}$ is defined by

$$
\operatorname{dict}_{d}(p):=x
$$


where $x$ is such that $p(d)=x \ldots$. So, $\operatorname{dict}_{d}(p)$ is the most preferred alternative of agent $d$ in $p(d)$. A social choice function $f$ is called nondictatorial if there is no agent $d$ such that $f=\operatorname{dict}_{d}$.

\section{Manipulation of social choice functions}

We are interested in strategic behaviour of individuals when facing cooperative decision-making as captured by social choice functions. This is formalized by the following definitions. Let $f: P^{N} \rightarrow A$ be a social choice function. Let $p \in P^{N}$ be a profile. Then each profile $q$ such that, for some $i \in N,\left.q\right|_{N-\{i\}}=\left.p\right|_{N-\{i\}}$ and $q(i) \neq p(i)$, is called an $i$-devation from $p$. Letting $t:=q(i)$ we use the notation $q=\left(p_{-i}, t\right)$. If it is not important which agent deviates from $p$ to $q$ we call $q$ a deviation from $p$. A profile $p$ is called manipulable (under $f$ ) if there is an agent that is better off by being dishonest about his preference, i.e. if there is an $i \in N$, and an $i$-deviation $q$ such that

$$
(f(p), f(q)) \notin p(i) .
$$

In this case we say that $p$ is manipulable towards $q$ (under $f$ ). Let $B \subseteq P^{N}$ and

$$
\begin{aligned}
M_{f}(B):= & \{p \in B \mid p \text { is manipulable towards } \\
& \text { some } q \in B \text { under } f\} .
\end{aligned}
$$

A social choice function is called strategy-proof on $B$ if $M_{f}(B)=\emptyset$, otherwise it is said to be manipulable on $B$. When $B=P^{N}$ we omit $B$ and write $M_{f}:=M_{f}\left(P^{N}\right)$.

The prominence of the dictatorial rules arises from the following impossibility result due to Gibbard (1973) and Satterthwaite (1975).

Theorem 1 Let $A$ be a finite set of alternatives, $|A| \geq 3$. Let $f: P^{N} \rightarrow A$ be a nondictatorial social choice function. Then

$$
\left|M_{f}\right| \geq 1 \text {. }
$$

However, this theorem makes no statement about the number of manipulable profiles such social choice functions admit. This question has been solved by Kelly (1988) for two agents and three alternatives, and by Fristrup and Keiding (1998) for two agents, and any number of alternatives larger than three. In Maus et al. (2004) it is shown that for $m=3$ and any number of agents larger than two, the following social choice functions are exactly the minimally manipulable ones among the nondictorial social choice functions. 
Definition 2 Choose $b, c \in A, b \neq c$, and let $A=\left\{a_{1}, \ldots a_{m-2}, b, c\right\}$. Choose $m-2$ different preferences $t_{j} \in P, j \in\{1,2, \ldots, m-2\}$, such that $t_{j}=$ $\ldots a_{j} \ldots b \ldots c \ldots$ for all $j \in\{1,2, \ldots, m-2\}$. Let $m: P^{N} \rightarrow A$ be the social choice function given by

$$
m(p):= \begin{cases}a_{j} & \text { if } p=t_{j}^{N} \text { for some } j \in\{1,2, \ldots, m-2\} \\ b & \text { if }(b, c) \in p(i) \text { for all } i \in N \text { and } p \notin\left\{t_{1}^{N}, \ldots t_{m-2}^{N}\right\} \\ c & \text { if there is an } i \in N \text { such that }(c, b) \in p(i) .\end{cases}
$$

Note that the social choice functions given by Definition 2 are not only nondictatorial but even anonymous. So, they are the minimally manipulable anonymous social choice functions for three alternatives. Hence, we do not have to treat the three alternative case here. We will show that also for $m \geq 4$ the social choice functions given by definition 2 are the minimally manipulable anonymous social choice functions. One might hope that as in the case $m=3$ the minimally manipulable nondictatorial social choice functions coincide with the anonymous ones. We will see that this is not so.

\section{Monotonicity and Pareto optimality on $B^{k}$}

Let $k \in \mathbb{N}$ and let

$$
B^{k}:=\left\{p \in P^{N}|| p^{-1}(N) \mid \geq k\right\}
$$

be the set of all profiles that contain at least $k$ different preferences. We want to show an impossibility result for strategy-proof and anonymous social choice functions on $B^{k}$ for certain $k$. This section contains two ingredients for that impossibility result, a monotonicity lemma and a Pareto optimality lemma.

Let $p, q \in P^{N}, a \in \widetilde{A} \subseteq A$. We say that a weakly improved from $p$ to $q$ with respect to $\widetilde{A}$, if for all alternatives $x \in \widetilde{A}$, we have for all agents $i \in N$

$$
(a, x) \in p(i) \text { implies }(a, x) \in q(i) \text {. }
$$

Let $f: P^{N} \rightarrow A$ be a social choice function and let $B \subseteq P^{N}$. We say that $f$ is monotone on $B$, if for all $p, q \in B$ such that $f(p)$ weakly improved from $p$ to $q$ with respect to $f(B)$, we have

$$
f(p)=f(q) .
$$

This monotonicity condition corresponds to the one used in Dasgupta et al. (1979) for social choice rules, and is also known as Maskin-monotonicity. The following lemma links strategy-proofness to monotonicity. 
Lemma 3 Let $f: P^{N} \rightarrow A$ be anonymous and strategy-proof on $B^{k}, k \in \mathbb{N}$. Then $f$ is monotone on $B^{k}$.

Proof. Let $p, q \in B^{k}$ be such that $f(p)$ weakly improves from $p$ to $q$ with respect to $f\left(B^{k}\right)$. We have to show that $f(p)=f(q)$. Let the profiles $r^{t} \in P^{N}, t \in\{0, \ldots, n\}$, be given by

$$
r^{t}(i):= \begin{cases}q(i) & i \leq t \\ p(i) & i>t\end{cases}
$$

for all $i \in N$. Then $r^{0}=p$ and $r^{n}=q$. For the moment assume that $r^{t} \in B^{k}$ for all $t \in\{0, \ldots, n\}$. As $f$ is strategy-proof on $B^{k}$ we have $\left(f\left(r^{1}\right), f\left(r^{0}\right)\right) \in$ $r^{1}(1)=q(1)$ and $\left(f\left(r^{0}\right), f\left(r^{1}\right)\right) \in p(1)$. As $f\left(r^{0}\right)=f(p)$ and $f(p)$ weakly improved from $p$ to $q$ with respect to $f\left(B^{k}\right),\left(f\left(r^{0}\right), f\left(r^{1}\right)\right) \in p(1)$ implies $\left(f\left(r^{0}\right), f\left(r^{1}\right)\right) \in q(1)$. So,

$$
\left(f\left(r^{1}\right), f\left(r^{0}\right)\right) \in r^{1}(1)=q(1)
$$

and

$$
\left(f\left(r^{0}\right), f\left(r^{1}\right)\right) \in q(1) .
$$

This implies that $f(p)=f\left(r^{0}\right)=f\left(r^{1}\right)$, since preferences are antisymmetric. Repeating this argument yields

$$
f(p)=f\left(r^{1}\right)=f\left(r^{2}\right)=\ldots=f\left(r^{n}\right)=f(q) .
$$

It remains to be shown that $r^{t} \in B^{k}$ for all $t \in\{0, \ldots, n\}$. Let

$$
v:=|p(N)|, w:=|q(N)| \text { and } u:=|q(N) \cap p(N)| .
$$

By anonymity, the order of the agents is not essential. We assume that the preferences in $p$ and $q$ are held by the agents in such a way that $|p(\{1, \ldots, v\})|=$ $v,|q(\{1, \ldots, w\})|=w$ and $p(i)=q(i)$ for all $i \in\{1,2, \ldots, u\}$. Now, if $l \in\{0,1, \ldots, u\}$, then $r^{l}=p \in B^{k}$ and if $l>\min \{v, w\}$ then $r^{l} \in B^{\min \{v, w\}}$, where $\min \{v, w\} \geq k$, so $r^{l} \in B^{k}$. In the remaining cases, i.e. if $u<l \leq$ $\min \{v, w\}$, we have $\left|r^{l}(N)\right| \geq\left|r^{l-1}(N)\right|$ as $r^{t}(l)=q(l) \notin q(\{1, \ldots, l-1\})=$ $r^{l}(\{1, \ldots, l-1\})$ and $r^{l}(l)=q(l) \notin\{p(\{l+1, \ldots, n\})$ by the way the preferences in $p$ and $q$ were arranged among the agents. Hence, $r^{l} \in B^{k}$ by a simple inductive reasoning based on $r^{u} \in B^{k}$.

As a corollary of Lemma 3 we can state the following.

Corollary 4 Let $f: P^{N} \rightarrow A$ be anonymous and strategy-proof on $B^{k}, k \in$ $\mathbb{N}$. Let $a \in f\left(B^{k}\right)$ and $p \in B^{k}$ be such that $(x, a) \notin p(i)$ for all $i \in N$ and all $x \in f\left(B^{k}\right)-\{a\}$. Then $f(p)=a$. 
Let $x, y \in A, x \neq y$. We say that $x$ Pareto dominates $y$ at the profile $p$, if $(x, y) \in p(i)$ for all $i \in N$. A social choice function is called Pareto optimal on $B \subseteq P^{N}$, if it does not choose alternatives Pareto dominated by an element of $f(B)$, i.e. there is no $x \in f(B)-\{f(p)\}$ such that $x$ Pareto dominates $f(p)$ at $p$.

The second lemma shows that if $f$ is anonymous and strategy-proof on $B^{k}$, then $f$ is Pareto optimal on $B^{k}$. We have to make restrictions on $k$ in order to make sure that there are sufficiently many different preferences having the same alternative at the first and second places.

Lemma 5 For $m=4$ let $k \leq m+1$ and for $m \geq 5$ let $k \leq m+2$. Let $f: P^{N} \rightarrow A$ be strategy-proof and anonymous on $B^{k}$. Then $f$ is Pareto optimal on $B^{k}$.

Proof. Let $p \in B^{k}, x \in f\left(B^{k}\right)$ and $y \in A$ be such that $x$ Pareto dominates $y$ at $p$. Suppose that $f(p)=y$. It is sufficient to deduce a contradiction. By Lemma $3 f$ is monotone on $B^{k}$. As $p \in B^{k}$ and $f$ is anonymous we can without loss of generality suppose that $|p(\{1, \ldots, k\})|=k$. Let $l:=$ $\max \{k-(m-2)$ !, 0$\}$, then by monotonicity we can assume that for $l<i \leq k$ we have $p(i)=x y \ldots$. In view of Corollary $4, l \geq 1$. For $i \in\{1, \ldots, l\}$ let $Z_{i}:=\{a \in A \mid(a, y) \in p(i)\}$ be the upper contour of $y$ at $p(i)$. There are precisely $(m-2)$ ! preferences where $x$ is best and $y$ is second best and $l \geq 1$, hence $k>(m-2)$ ! and $\{x, y\} \subsetneq Z_{i}$ for all $i \in\{1, \ldots, l\}$. Consider $r(i)$ obtained from $p(i)$ by shifting alternative $x$ to the top leaving all other alternatives unchanged:

$$
r(i)=(\{x\} \times A) \cup\left(p(i) \cap(A-\{x\})^{2}\right) .
$$

For $l<i \leq k$ we have $r(i)=p(i)$. If we show that $r \in B^{k}$ then by monotonicity $f(r)=y$, as $y=f(p)$ improves from $p$ to $r$.

Case 1: $\left|\left\{Z_{1}, \ldots, Z_{l}\right\}\right|=l$.

As $\{x, y\} \subsetneq Z_{i}$ and $\left|\left\{Z_{1}, \ldots, Z_{l}\right\}\right|=l,|r(\{1, \ldots, k\})|=k$. Hence, $r \in B^{k}$. By Corollary $4 f(r)=x$, contradicting $f(r)=y$.

Case 2: $\left|\left\{Z_{1}, \ldots, Z_{l}\right\}\right|<l$.

So, $l \geq 2$. But $l=k-(m-2) ! \leq(m+2)-(m-2) ! \leq 1$ for $m \geq 5$. So, $m=4$ and $l \in\{2,3\}$. Without loss of generality $Z_{1}=Z_{2}$ and let $A=\{x, y, a, b\}$. As $\{x, y\} \subsetneq Z_{i}$ for all $i \in\{1, \ldots, l\}$, there are alternatives $z_{1} \in Z_{1}-\{x, y\}$ and, if $l=3, z_{3} \in Z_{3}-\{x, y\}$. If $l=3$ and $Z_{3}=Z_{1}=Z_{2}$ we must have $\left|Z_{1}\right|=4$ as $\left|Z_{1}\right|=3$ implies that without loss of generality $p(\{1,2,3\}) \subseteq\{a x y b, x a y b\}$, contradicting $|p(\{1, \ldots, k\})|=k$. So, if $l=3$, we can choose a $z_{3} \in Z_{1}-\left\{x, y, z_{1}\right\}$. Without loss of generality suppose that 
$z_{1}=a$ and, if $l=3, z_{3}=b$. Consider the profile $\bar{r}$ defined by

$$
\bar{r}(i):= \begin{cases}a x y b & \text { if } i=1, \\ x a y b & \text { if } i=2, \\ x b y a & \text { if } i=l=3 \\ r(i) & \text { if } i>l\end{cases}
$$

Then $\bar{r} \in B^{k}$ and $y$ improves from $p$ to $\bar{r}$. Hence, $f(\bar{r})=y$. Let $\widehat{r}:=$ $\left(r_{-1}, x a b y\right)$. By Lemma $4, f(\widehat{r})=x$. So, $\widehat{r}$ is a 1-deviation from $\bar{r}$ such that $(f(\bar{r}), f(\widehat{r})) \notin \bar{r}(1)$. But then $\bar{r} \in B^{k}$ is manipulable and this contradiction ends the proof.

\section{Decisiveness on $B_{\{a, b\}}^{k}$}

Let $X \subseteq A$ and $B_{X}^{k}$ be the set of all profiles in $B^{k}$ where all alternatives in $X$ are strictly preferred to alternatives in $A-X$,

$B_{X}^{k}:=\left\{p \in B^{k} \mid\right.$ for all $i \in N$ and $x \in X, y \in A-X$ we have $\left.(x, y) \in p(i)\right\}$.

Let $p \in B_{\{a, b\}}^{k}, a \neq b$. We say that $S \subseteq N$ decides over $(a, b)$ at $p$, if $f(p)=a$ and $S=\{i \in N \mid(a, b) \in p(i)\}$. We say that $S$ decides over $(a, b)$, if $S$ decides over $(a, b)$ at all profiles $p \in B_{\{a, b\}}^{k}$ that satisfy $S=\{i \in N$ | $(a, b) \in p(i)\}$. We denote the set of all $S$ that are decisive over $(a, b)$ by $W^{k}(a, b)$. The following is an immediate consequence of the definitions of weak improvement and monotonicity.

Remark 6 For social choice functions $f$ which are monotone on $B^{k}, S$ decides over $(a, b)$ at a profile $p \in B_{\{a, b\}}^{k}$ if and only if $S \in W^{k}(a, b)$.

The next lemma shows some decisiveness properties of $f$ on $B^{k}$ over triples of alternatives, when $f$ is strategy-proof on $B^{k}$.

Lemma 7 Let $k \leq m$ if $m=4$ and $k \leq m+2$ if $m \geq 5$. Let $f$ be anonymous and strategy-proof on $B^{k}, f\left(B^{k}\right) \supseteq\{a, b, c\}$ for different $a, b$ and c. Let $S \in W^{k}(a, b)$. Then

1. $S \in W^{k}(c, b)$ and $S \in W^{k}(a, c)$,

2. $S \in W^{k}(b, a)$ and

3. $|S|>\frac{1}{2} n$. 
Proof. (1): By Lemma $3 f$ is monotone on $B^{k}$. By the restrictions on $k$ $B_{\{a, b\}}^{k}$ is nonempty. Let $p \in B_{\{a, b\}}^{k}$ be such that $S$ is decisive over $(a, b)$ at $p$. For a permutation $\tau$ on $A$ and a preference $t \in P$ let

$$
t^{\tau}=\{(\tau(x), \tau(y)) \mid(x, y) \in t\} .
$$

For $x, y \in A$ let $(x y)$ denote the permutation that maps $x$ on $y, y$ on $x$ and all other elements of $A$ on themselves.

Let $p^{1}$ be such that $\left.p^{1}\right|_{S}=\left.p\right|_{S}$ and $p^{1}(i)=(p(i))^{(a c)}$ for all $i \in N-S$. Then $p^{1} \in B^{k}$. By monotonicity $f\left(p^{1}\right) \neq b$, as $b$ weakly improves from $p^{1}$ to $p$ and $f(p)=a \neq b$. As $b$ Pareto dominates all $x \in A-\{a, b\}$ at $p^{1}$ we also have by Lemma 5 that $f\left(p^{1}\right) \notin A-\{a, b\}$, so $f\left(p^{1}\right)=a$.

Let $p^{2}$ be such that $\left.p^{2}\right|_{N-S}=\left.p^{1}\right|_{N-S}$ and $p^{2}(i)=\left(p^{1}(i)\right)^{(b c)}$ for all $i \in S$. Then $p^{2} \in B^{k}$. By monotonicity $f\left(p^{2}\right)=a$, as $a$ weakly improves from $p^{1}$ to $p^{2}$ and $f\left(p^{1}\right)=a$.

Let $p^{3}$ be such that $\left.p^{3}\right|_{N-S}=\left.p^{2}\right|_{N-S}$ and $p^{3}(i)=\left(p^{2}(i)\right)^{(a c)}$ for all $i \in S$. Then $p^{3} \in B^{k}$. As $c \in f\left(B^{k}\right)$ Pareto dominates all alternatives $x \in A-\{b, c\}$ it follows by Lemma 5 that $f\left(p^{3}\right) \in\{b, c\}$. By monotonicity $f\left(p^{3}\right) \neq b$, as $b$ weakly improves from $p^{3}$ to $p^{2}$ and $f\left(p^{2}\right)=a \neq b$. So, $f\left(p^{3}\right)=c$.

Finally, let $p^{4}$ be such that $\left.p^{4}\right|_{N-S}=\left.p^{3}\right|_{N-S}$ and $p^{4}(i)=\left(p^{3}(i)\right)^{(a b)}$ for all $i \in S$. Then $p^{4} \in B_{\{b, c\}}^{k}$ and by monotonicity $f\left(p^{4}\right)=c$. So, $S$ decides over $(c, b)$ at $p^{4}$ and by Remark 6

$$
S \in W^{k}(c, b) .
$$

In order to see that $S$ decides over $(a, c)$ let $p^{5}$ be such that $\left.p^{5}\right|_{S}=\left.p^{2}\right|_{S}$ and $p^{5}(i)=\left(p^{2}(i)\right)^{(b c)}$ for all $i \in N-S$. Then $p^{5} \in B^{k}$ and as $f\left(p^{2}\right)=a$ we have by monotonicity that $f\left(p^{5}\right)=a$.

Let $p^{6}$ be such that $\left.p^{6}\right|_{S}=\left.p^{5}\right|_{S}$ and $p^{6}(i)=\left(p^{5}(i)\right)^{(a b)}$ for all $i \in N-S$. Then $p^{6} \in B_{\{a, c\}}^{k}$ and by monotonicity $f\left(p^{6}\right)=a$. So, $S$ decides over $(a, c)$ at $p^{6}$ and by Remark 6

$$
S \in W^{k}(a, c) .
$$

(2): By (1) we have $S \in W^{k}(a, c)$. Thus, applying (1) to $S \in W^{k}(a, c)$ yields $S \in W^{k}(b, c)$ and then applying the second part of (1) to $S \in W^{k}(b, c)$ yields $S \in W^{k}(b, a)$.

(3): To the contrary suppose that $|S| \leq \frac{1}{2} n$. By anonymity we have $T \in \bar{W}^{k}(a, b)$ for all $T$ such that $|T|=|S|$. By $(2)$ also $T \in W^{k}(b, a)$ for all such $T$. By monotonicity then $T \in W^{k}(b, a)$ for all $T$ such that $|T| \geq|S|$. So, $N-S \in W^{k}(b, a)$ and this contradicts $S \in W^{k}(a, b)$.

In the case $m=4$ and $k=m+1=5, B_{\{a, b\}}^{k}$ is empty, but an almost similar result can be achieved using the following terminology. Let $A=$ 
$\{a, b, c, d\}$. Let $B_{\{a, b\}}^{k, d}$ be the set of all profiles $p$ in $B^{k}$ where all except one agent $i \in N$ prefer all alternatives in $\{a, b\}$ to all alternatives in $\{c, d\}$, and $p(i) \in\{a d b c, b d a c\}$. Let $p \in B_{\{a, b\}}^{k, d}, a \neq b$. We say that $S \subseteq N d$-decides over $(a, b)$ at $p$, if $f(p)=a$ and $S=\{i \in N \mid(a, b) \in p(i)\}$, adbc $\notin p(S)$. We say that $S d$-decides over $(a, b)$, if $S d$-decides over $(a, b)$ at all profiles $p \in B_{\{a, b\}}^{k, d}$ that satisfy $S=\{i \in N \mid(a, b) \in p(i)\}, a d b c \notin p(S)$. We denote the set of all $S$ that are $d$-decisive over $(a, b)$ by $W_{d}^{k}(a, b)$.

As before we make the following observation.

Remark 8 For social choice functions $f$, which are monotone on $B^{k}, S d$ decides over $(a, b)$ at a profile $p \in B_{\{a, b\}}^{k, d}$ if and only if $S \in W_{d}^{k}(a, b)$.

Now we can show a lemma like Lemma 7 for $d$-decisiveness.

Lemma 9 Let $A=\{a, b, c, d\}$ and $k=5$. Let $f$ be anonymous and strategyproof on $B^{k}, f\left(B^{k}\right) \supseteq\{a, b, c\}$ for pairwise different $a, b$ and $c$. Let $S \in$ $W_{d}^{k}(a, b)$. Then
1. $S \in W_{d}^{k}(c, b)$ and $S \in W_{d}^{k}(a, c)$
2. $S \in W_{d}^{k}(b, a)$
3. $|S| \geq \frac{1}{2} n$.

Proof. By Lemma $3 f$ is monotone on $B^{k}$. Let $S \in W_{d}^{k}(a, b)$. Then $|p(S)|=2$ by definition, and thus $2 \leq|S| \leq n-(k-|p(S)|)=n-3$. Without loss of generality $S=\{1, \ldots,|S|\}$. Consider the following profiles.

\begin{tabular}{|c|c|c|c|c|c|c|c|}
\hline & $p$ & $p^{1}$ & $p^{2}$ & $p^{3}$ & $p^{4}$ & $p^{5}$ & $p^{6}$ \\
\hline$\{1\}$ & $a b c d$ & $a b c d$ & $a c b d$ & $c a b d$ & $c b a d$ & $a c b d$ & $a c b d$ \\
\hline$S-\{1\}$ & $a b d c$ & $a b d c$ & $a c d b$ & $c a d b$ & $c b d a$ & $a c d b$ & $a c d b$ \\
\hline$N-\{n-1, n\}$ & $b a c d$ & $b c a d$ & $b c a d$ & $b c a d$ & $b c a d$ & $c b a d$ & $c a b d$ \\
\hline$n-1$ & $b a d c$ & $b c d a$ & $b c d a$ & $b c d a$ & $b c d a$ & $c b d a$ & $c a d b$ \\
\hline$n$ & $b d a c$ & $b d c a$ & $b d c a$ & $b d c a$ & $b d c a$ & $c d b a$ & $c d a b$ \\
\hline
\end{tabular}

As in the proof of Lemma 7 by these profiles it follows that $S \in W_{d}^{k}(c, b)$ and $S \in W_{d}^{k}(a, c)$. Then (2) follows by applying (1) twice as in the proof of Lemma 7. Furthermore, if $T \subseteq N$ such that $|T| \geq S$ and $|T| \leq n-3$, then by anonymity and monotonicity $T \in W_{d}^{k}(b, a)$. Suppose $S \in W_{d}^{k}(a, b)$ and $|S|<\frac{1}{2} n$. Then $|N-(S \cup\{n\})|=|N|-|S|-1>\frac{1}{2} n-1 \geq|S|$. So, $N-(S \cup\{n\}) \in W_{d}^{k}(b, a)$. Consider the profile $q=\left(p_{-n}, a d b c\right)$. By strategyproofness $f(q)=a$. But $\{i \in N \mid(b, a) \in q(i)\}=N-(S \cup\{n\}) \in W_{d}^{k}(b, a)$, so $f(q)=b$, a contradiction. 


\section{Gibbard-Satterthwaite on $B^{k}$}

We combine the results of the previous sections to show an impossibility result for strategy-proof anonymous social choice functions among more than two alternatives on the restricted domain $B^{k}$, i.e. when agents preferences have to satisfy a minimal diversity.

Theorem 10 If $m=4$ let $k \leq m+1 \leq n$ and if $m \geq 5$ let $k \leq m+2 \leq n$. Let $f$ be strategy-proof on $B^{k}$ and anonymous. Then $\left|f\left(B^{k}\right)\right| \leq 2$.

Proof. To the contrary suppose that there are pairwise different $a, b, c \in$ $f\left(B^{k}\right)$. Note that by the constraints $n \geq 5$. By Lemma $3 f$ is monotone on $B^{k}$. Let $\{S, T, U\}$ be a partition of $N$, such that for $X, Y \in\{S, T, U\}$ we have $0<|X| \leq|Y|+1$. Consider profiles $p$ such that

$$
\begin{aligned}
& p(S) \subseteq\{t \in P \mid t=a b \ldots\}, \\
& p(T) \subseteq\{t \in P \mid t=b c \ldots\} \text { and } \\
& p(U) \subseteq\{t \in P \mid t=c a \ldots\} .
\end{aligned}
$$

There are $(m-2)$ ! different choices for any $p(i)$, hence we may assume that $p \in B^{k}$. Without loss of generality $f(p)=a$. For some $\widetilde{S}$ such that $|\widetilde{S}|<\frac{1}{2} n$ we will prove that $\widetilde{S} \in W^{k}(a, c)$ if $(m, k) \neq(4,5)$ and $\widetilde{S} \in W_{d}^{k}(a, c)$ if $(m, k)=(4,5)$. As $|\widetilde{S}|<\frac{1}{2} n$, we have a contradiction with Lemma 7 , or Lemma 9 respectively, and are done.

Case 1: $(m, k) \neq(4,5)$

Let $\{\widetilde{S}, \widetilde{T}, \widetilde{U}\}$ be a partition of $N$ such that $S \subseteq \widetilde{S}, T \supseteq \widetilde{T}, U \supseteq \widetilde{U}$ and for $X, Y \in\{\widetilde{S}, \widetilde{T}, \widetilde{U}\}$ we have $0<|X| \leq \min \{|Y|+1,|\widetilde{S}|\}$. Consider $q$ such that

$$
\begin{aligned}
q(\widetilde{S}) & \subseteq\{t \in P \mid t=a c \ldots\}, \\
q(\widetilde{T}) & \subseteq\{t \in P \mid t=c a \ldots\} \text { and } \\
q(\widetilde{U}) & \subseteq\{t \in P \mid t=c a \ldots\} .
\end{aligned}
$$

A preference $q(i)$ in $\widetilde{S}$ or $\widetilde{T} \cup \widetilde{U}$ can be extended in $(m-2)$ ! ways. We prove that there are such $q \in B_{\{a, c\}}^{k}$. So, we have to show that

$$
k \leq \min \{|\widetilde{S}|,(m-2) !\}+\min \{|\widetilde{T}|+|\widetilde{U}|,(m-2) !\}=: \alpha .
$$

There are four cases. If $\alpha=|\widetilde{S}|+|\widetilde{T}|+|\widetilde{U}|=|N|$ then $\alpha=n \geq k$. If $\alpha=|\widetilde{S}|+(m-2)$ !, then, as $|\widetilde{S}| \geq 2$,

$$
\alpha \geq 2+(m-2) ! \geq m+2 \geq k
$$


if $m \geq 5$, and

$$
\alpha \geq 2+(m-2) !=m \geq k
$$

if $m=4$, since $(m, k) \neq(4,5)$. If $\alpha=2(m-2)$ !, then $\alpha \geq 2+(m-2)$ ! and we can argue as in the previous case. As $n \geq m+1$ and $m \geq 4, n \geq 5$. So, $|\widetilde{S}|<|\widetilde{T}|+|\widetilde{U}|$. Therefore the case $\alpha=(m-2) !+\widetilde{T}|+| \widetilde{U} \mid$ cannot occur. So, we may assume that $q \in B_{\{a, c\}}^{k}$.

From $p$ to $q a$ weakly improves, so by monotonicity $f(q)=a$, and therefore $\widetilde{S} \in W^{k}(a, c)$. By the way $\widetilde{S}$ was chosen we have $|\widetilde{S}|<\frac{1}{2} n$, contradicting part 3 of Lemma 7.

Case 2: $(m, k)=(4,5)$

Subcase $2^{A}:|S|=1$

Then $n=5$, and by anonymity we may assume that

$$
p=(a c \ldots, b c a d, b c d a, c a b d, c a d b) \text {. }
$$

Let

$$
q=(a c d b, a c b d, c d a b, c a b d, c a d b) .
$$

Then $a$ has improved from $p$ to $q$, so $f(q)=a$ by monotonicity. But then $\{i \in N \mid(a, c) \in q(i)\}=S=\{1,2\} \in W_{d}^{k}(a, c)$, contradicting part 3 of Lemma 9 .

$\underline{\text { Subcase } 2^{B}:}|S| \geq 2$

Let $p^{1}$ be such that $\left.p^{1}\right|_{N-S}=\left.p\right|_{N-S}$ and $p^{1}(i)=(p(i))^{(b c)}$ for all $i \in S$. Then $p^{1} \in B^{k}$. By monotonicity $f\left(p^{1}\right)=a$, as $a$ weakly improves from $p$ to $p^{1}$. Without loss of generality $1 \in T$. Let $p^{2}$ be equal to $p^{1}$ if there is an agent $\widehat{i} \in T$ with $p^{1}(i)=b c d a$. So, $f\left(p^{2}\right)=a$. Otherwise $p^{1}(T)=\{b c a d\}$ and we let $p^{2}:=\left(p_{-\hat{i}}, b c d a\right)$ for some $\hat{i} \in T$. Obviously $p^{2} \in B^{5}$. Now $c$ Pareto dominates $d$ at $p^{2}$, so $d \neq f\left(p^{2}\right)$ by Lemma 5 . As $f$ is strategy-proof on $B^{5}$ and $f\left(p^{2}\right) \notin\{b, c\}$ we obtain $f\left(p^{2}\right)=a$ in the case $p^{1}(T)=\{b c a d\}$ as well. Now consider $p^{3}$ such that $\left.p^{3}\right|_{N-T}=\left.p^{2}\right|_{N-T}, p^{3}(1)=c d a b$ and $p^{3}(T-\{1\})=\{c a b d\}$. Then $a$ weakly improves from $p^{2}$ to $p^{3}$, and $p^{3} \in B^{k}$, so by monotonicity $f\left(p^{3}\right)=a$. But then $\left\{i \in N \mid(a, c) \in p^{3}(i)\right\}=S \in W_{d}^{k}(a, c)$, contradicting part 3 of Lemma 9 .

The theorem says that within the restrictions the impossibility result of Gibbard Satterthwaite holds already on the smaller domain of profiles that satisfy a certain diversity. This may not surprise the reader because practically situations with a lot of diversity can be expected to be suspect to manipulation. At least it confirms this expectation. We did not think much about the situation when $k$ exceeds the bounds given. This is beyond the scope of this paper since the result is only a by-product on the way to the main result contained in the next section. 


\section{Minimal manipulability of anonymous so- cial choice functions}

Before proving the main result we show some helpful inequalities.

Lemma 11 Let $k, l, m, n \in \mathbb{N}$. Then

1. $k ! l ! \leq(k+l-1) !$

2. If $2 \leq k \leq m+1 \leq n$ and $m \geq 4$, then

$$
\left(\frac{m !}{3}-k\right) \frac{(n-1) !}{(n-k) !}>\left(\frac{m !}{3}-1\right)(m-2) .
$$

Proof. (1):

$$
k ! l ! \leq(k+l-1) ! \Longleftrightarrow k+l \leq\left(\begin{array}{c}
k+l \\
k
\end{array}\right) \Longleftrightarrow k, l \geq 1
$$

(2): Note that

$$
\begin{gathered}
\left(\frac{m !}{3}-k\right) \frac{(n-1) !}{(n-k) !}>\left(\frac{m !}{3}-1\right)(m-2) \\
\Longleftrightarrow \quad\left(\frac{m !}{3}-k\right)\left(\frac{(n-1) !}{(n-k) !(m-2)}\right)-\frac{m !}{3}>-1 \\
\Longleftrightarrow \quad \frac{m !}{3}\left(\left(\frac{(n-1) !}{(n-k) !(m-2)}\right)-1\right)>k\left(\frac{(n-1) !}{(n-k) !(m-2)}\right)-1
\end{gathered}
$$

Let $\alpha:=\frac{(n-1) !}{(n-k) !(m-2)}$. Then we have to show that $\frac{m !}{3}(\alpha-1)>k \alpha-1$.

Now, for $m \geq 5$ we have $m ! \geq 3(m+1)^{2}$, so it is sufficient to prove that $(m+1)^{2}(\alpha-1) \geq k \alpha$. In view of $k \leq m+1$ it is then enough to show that $(m+1) \alpha-1 \geq \alpha \Longleftrightarrow m \alpha>1$ which is satisfied, as clearly $\alpha>\frac{1}{m-2}$. If $m=4$ then we have to show that $8(\alpha-1)>k \alpha-1 \Longleftrightarrow(8-k) \alpha>7$.

If $k=2$ then this becomes $6 \frac{(n-1)}{2}>7$, which is satisfied as $n \geq 5$. If $2<k \leq 5$ then also $(8-k) \alpha>3 \frac{(n-1)(n-2)}{2}>7$, as $n \geq 5$. This proves (2).

Now we can prove the main result. It characterizes the minimally manipulable anonymous social choice functions for more than three alternatives.

Theorem 12 Let $f: P^{N} \rightarrow A, m \geq 4$, be an anonymous social choice function. Suppose that $n \geq m+1$ if $m=4$ and that $n \geq m+2$ if $m \geq 5$. Then

$$
\left|M_{f}\right| \geq n\left(\frac{m !}{3}-1\right)(m-2) .
$$

Furthermore, equality holds if and only if $f$ can be described as in definition 2. 
Proof. Let $P=\left\{t_{1}, \ldots, t_{m !}\right\}$ and $0 !=1$. Let $p \in P^{N}$ and let $c(p, i):=$ $\left|p^{-1}\left(t_{i}\right)\right|, i \in\{1, \ldots, m !\}$. Then there are

$$
\frac{n !}{\prod_{i=1}^{m !} c(p, i) !}
$$

profiles $q \in P^{N}$ that satisfy $c(q, i)=c(p, i)$ for all $i \in\{1, \ldots, m !\}$. Under anonymity $f(p)=f(q), p \in M_{f} \Longleftrightarrow q \in M_{f}$ and $p \in B^{k} \Longleftrightarrow q \in B^{k}$ for all such profiles $q$. Let

$$
l:= \begin{cases}m+1 & \text { if } m=4 \\ m+2 & \text { if } m \geq 5\end{cases}
$$

Suppose that the assumptions of the theorem are satisfied, and that

$$
\left|M_{f}\right| \leq n\left(\frac{m !}{3}-1\right)(m-2) .
$$

Step 1: $B^{l} \cap M_{f}=\emptyset$.

Suppose to the contrary that there is a $p \in B^{l} \cap M_{f}$. As $p \in B^{l}$, at least $l$ of the $c(p, i), i \in\{1, \ldots, m !\}$, satisfy $c(p, i) \geq 1$. Hence, by Lemma 11

$$
\prod_{i=1}^{m !} c(p, i) ! \leq\left(\sum_{i=1}^{m !} c(p, i)-l\right) !=(n-l) !
$$

So,

$$
\left|M_{f}\right| \geq \frac{n !}{\prod_{i=1}^{m !} c(p, i) !} \geq \frac{n !}{(n-l) !}=n \alpha(l),
$$

where $\alpha(l):=\frac{(n-1) !}{(n-l) !}$.

Now, if $m=4$, then as $n \geq m+1$,

$$
\begin{aligned}
\alpha(l) & =\prod_{j=1}^{l-1}(n-j) \geq \prod_{j=1}^{m}(m+1-j)=m ! \\
& =3 \frac{m !}{3}>2\left(\frac{m !}{3}-1\right)=(m-2)\left(\frac{m !}{3}-1\right),
\end{aligned}
$$

and, if $m \geq 5$, then, as $n \geq m+2$,

$$
\begin{aligned}
\alpha(l) & =\prod_{j=1}^{l-1}(n-j) \geq \prod_{j=1}^{m+1}(m+2-j)=(m+1) ! \\
& >(m+1)\left(\frac{m !}{3}-1\right)>(m-2)\left(\frac{m !}{3}-1\right) .
\end{aligned}
$$


This contradicts

$$
\left|M_{f}\right| \leq n(m-2)\left(\frac{m !}{3}-1\right)
$$

so step 1 is proven.

Step 2: There are $b, c \in A, b \neq c$, such that $f\left(B^{2}\right) \subseteq\{b, c\}$.

$\overline{\text { By step }} 1 f$ is strategy-proof on $B^{l}$. So, by Theorem $10,\left|f\left(B^{l}\right)\right| \leq 2$. Let $b, c \in A, b \neq c$, be such that $f\left(B^{l}\right) \subseteq\{b, c\}$. Let $k$ be the smallest number such that

$$
f\left(B^{k}\right) \subseteq\{b, c\} .
$$

Then $k \geq 2$ by surjectivity of $f$, and $k \leq l$ since $f\left(B^{l}\right) \subseteq\{b, c\}$. We show that $k=2$. As $f\left(B^{k-1}\right) \nsubseteq\{b, c\}$, there is a $p \in B^{k-1}-B^{k}$, such that $f(p)=a \in A-\{b, c\}$. Furthermore, as $k \leq n$, and $p \in B^{k-1}-B^{k}$, there must be agents $i, j \in N, i \neq j$, such that $p(i)=p(j)$, which implies that $p(N)=p(N-\{i\})$. Let $t$ be such that

$$
\{(a, c),(b, c)\} \subset t \text { and } t \notin p(N) .
$$

As $|p(N)|=k-1$, there are at least $\frac{m !}{3}-(k-1)$ such preferences $t$. Then $t \notin p(N)$ implies that $q=\left(p_{-i}, t\right) \in B^{k}$. So $f(q) \in\{b, c\}, f(p)=a$ and $(f(q), f(p)) \notin t$. Hence, any such $q$ is manipulable. So, by anonymity

$$
\left|M_{f}\right| \geq\left(\frac{m !}{3}-(k-1)\right) \frac{n !}{(n-(k-1)) !} .
$$

But, by Lemma 11 the term on the right hand side is greater than $n(m-$ $2)\left(\frac{m !}{3}-1\right)$ if $k-1 \geq 2$, contradicting $\left|M_{f}\right| \leq n(m-2)\left(\frac{m !}{3}-1\right)$. Therefore, $k=2$. This proves step 2 .

Let $a \in A$ be an alternative different from $b$ and $c$ as in step 2. We define

$$
P^{a,\{b, c\}}:=\{t \in P \mid\{(a, b),(a, c)\} \subseteq t\} .
$$

Let $A=\left\{a_{1}, a_{2}, \ldots, a_{m-2}, b, c\right\}$. As $f\left(B^{2}\right) \subseteq\{b, c\}$, we have

$$
f\left(P^{N}-B^{2}\right) \supseteq\left\{a_{1}, a_{2}, \ldots, a_{m-2}\right\}
$$

by surjectivity. Hence, for any $a_{j}, j \in\{1,2, \ldots, m-2\}$, there is a $t_{j} \in P$ such that $f\left(t_{j}^{N}\right)=a_{j}$. Let $p^{j}:=t_{j}^{N}, j \in\{1,2, \ldots, m-2\}$. Then for any $t \in P^{a_{j},\{b, c\}}-\left\{t_{j}\right\}, k \in N, f\left(p_{-k}^{j}, t\right) \in\{b, c\}$ and $f\left(p^{j}\right)=a_{j}$, so $q=\left(p_{-k}^{j}, t\right)$ is manipulable for all $k \in N$. There are

$$
n\left|P^{a_{j},\{b, c\}}-\left\{t_{j}\right\}\right|
$$


such $q$, as $n \geq 3$ these manipulable profiles $q$ are different for all $j \in$ $\{1,2, \ldots, m-2\}$. So,

$$
n(m-2)\left(\frac{m !}{3}-1\right) \geq\left|M_{f}\right| \geq n \sum_{i=1}^{m-2}\left|P^{a_{j},\{b, c\}}-\left\{t_{j}\right\}\right| .
$$

Now, $\left|P^{a_{j},\{b, c\}}-\left\{t_{j}\right\}\right| \geq \frac{m !}{3}-1$, and equality holds if and only if $t_{j} \in P^{a_{j},\{b, c\}}$. So, by inequality $(1), t_{j} \in P^{a_{j},\{b, c\}}, j \in\{1,2, \ldots, m-2\}$,

$$
\left|M_{f}\right|=n(m-2)\left(\frac{m !}{3}-1\right)
$$

and

$$
M_{f}=\left\{\left(p_{-k}^{j}, t\right) \mid k \in N, j \in\{1,2, \ldots, m-2\}, t \in P^{a_{j},\{b, c\}}-\left\{t_{j}\right\}\right\} .
$$

Step 3: Let $b, c$ be as in step 2. Then

$$
f\left(P^{N}-\left\{p^{1}, p^{2}, \ldots, p^{m-2}\right\}\right)=\{b, c\}
$$

Let $p \in P^{N}-\left\{p^{1}, p^{2}, \ldots, p^{m-2}\right\}$. Suppose that $f(p) \in\left\{a_{1}, a_{2}, \ldots, a_{m-2}\right\}$. Then $p \in P^{N}-B^{2}$ and for all $t \in P^{f(p),\{b, c\}}-\{p(1)\}$ we have $f\left(p_{-1}, t\right) \in$ $f\left(B^{2}\right) \subseteq\{b, c\}$. So $\left(p_{-1}, t\right) \in M_{f}$, implying that $p \in\left\{p^{1}, p^{2}, \ldots, p^{m-2}\right\}$, as $n \geq 3$. With surjectivity this proves step 3 .

Step 4: Let $p \notin\left\{p^{1}, p^{2}, \ldots, p^{m-2}\right\}$ and suppose that $(b, c) \in t_{j}$ for some

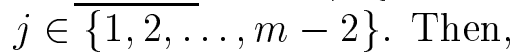

$$
f(p)=b \text { if and only if }(b, c) \in p(i) \text { for all } i \in N .
$$

Let $t, \bar{t} \in P$ be such that $t=\ldots b \ldots a_{j} \ldots c \ldots, \bar{t}=\ldots c \ldots a_{j} \ldots b \ldots$ Let $k \in N$. As $t, \bar{t} \notin P^{a_{j},\{b, c\}}, f$ is strategy-proof at $\left(p_{-k}^{j}, t\right) \in B^{2}$ and $\left(p_{-k}^{j}, \bar{t}\right) \in B^{2}$. This implies that $f\left(p_{-k}^{j}, t\right)=b$ and $f\left(p_{-k}^{j}, \bar{t}\right)=c$.

Suppose that there is a $i \in N$ such that $(c, b) \in p(i)$, without loss of generality $i=n$. Let

$$
r^{u}(l):=\left\{\begin{array}{cc}
p(l) & \text { if } l \leq u \\
\left(p_{-n}^{j}, \bar{t}\right)(l) & \text { if } l>u
\end{array}\right.
$$

$u \in\{0, \ldots, n\}$. Then $r^{0}=\left(p_{-n}^{j}, \bar{t}\right), r^{n}=p$ and as $\bar{t} \notin P^{a_{j},\{b, c\}}$,

$$
r^{u} \notin M_{f} \cup\left\{p^{1}, p^{2}, \ldots, p^{m-2}\right\}, u \in\{0, \ldots, n-1\} .
$$

Suppose that $f\left(r^{u}\right)=c, u \in\{0, \ldots, n-2\}$, then by $r^{u} \notin M_{f}$

$$
\left(f\left(r^{u}\right), f\left(r^{u+1}\right)\right) \in r^{u}(u+1)=t_{j} .
$$


As $(b, c) \in t_{j}, f\left(r^{u+1}\right) \in\{b, c\}$ and $f\left(r^{u}\right)=c$ it follows that $f\left(r^{u+1}\right)=c$. As $f\left(r^{0}\right)=f\left(p_{-n}^{j}, \bar{t}\right)=c$ we obtain $f\left(r^{n-1}\right)=f(p)=c$ by induction. By strategy-proofness at $p=r^{n}=\left(r_{-n}^{n-1}, p(n)\right),(c, b) \in p(n)$, and $f(p) \in\{b, c\}$ it follows that $f\left(r^{n}\right)=c$. This shows that

$$
f(p)=b \text { only if }(b, c) \in p(i) \text { for all } i \in N .
$$

Suppose that $(b, c) \in p(i)$ for all $i \in N$, so in particular $(b, c) \in p(1)$. Let

$$
r^{u}(l):=\left\{\begin{array}{cc}
\left(p_{-1}^{j}, t\right)(l) & \text { if } l \leq u \\
p(l) & \text { if } l>u
\end{array}\right.
$$

$u \in\{0, \ldots, n\}$. Then $r^{0}=p, r^{n}=\left(p_{-1}^{j}, t\right)$, and as $t \notin P^{a_{j},\{b, c\}}$,

$$
r^{u} \notin M_{f} \cup\left\{p^{1}, p^{2}, \ldots, p^{m-2}\right\}, u \in\{1, \ldots, n\} .
$$

As $r^{n}=\left(p_{-1}^{j}, t\right), f\left(r^{n}\right)=b$. Let $u \in\{1,2, \ldots, n\}$ and suppose that $f\left(r^{u}\right)=b$. Then by $r^{u} \notin M_{f}\left(f\left(r^{u}\right), f\left(r^{u-1}\right)\right) \in r^{u}(u)=\left(p_{-1}^{j}, t\right)(u) \in\left\{t, t_{j}\right\}$. As $(b, c) \in$ $t,(b, c) \in t_{j}, f\left(r^{u-1}\right) \in\{b, c\}$ and $f\left(r^{u}\right)=b$ it follows that $f\left(r^{u-1}\right)=b$. So, $f\left(r^{n}\right)=f\left(p_{-1}, t\right)=b$ implies by induction that $f\left(r^{0}\right)=f(p)=b$. This shows that

$$
f(p)=b \text { if }(b, c) \in p(i) \text { for all } i \in N,
$$

and we have proven step 4 .

Without loss of generality $(b, c) \in t^{1}$. Then step 4 and the well-definedness of $f$ imply that $(b, c) \in t_{j}$ for all $j \in\{1,2, \ldots, m-2\}$. This completes the proof.

Almost dictatorial social choice functions have $(n-1)\left(\frac{m !}{2}-1\right)+1$ manipulable profiles, see Maus et al. (2004) for a definition of almost dictatorial social choice functions and a proof of this statement. As, if $m \geq 4$,

$$
\begin{aligned}
n(m-2)\left(\frac{m !}{3}-1\right) & >n\left(\frac{m !}{2}-1\right) \\
& =(n-1)\left(\frac{m !}{2}-1\right)+\left(\frac{m !}{2}-1\right) \\
& >(n-1)\left(\frac{m !}{2}-1\right)+1,
\end{aligned}
$$

the minimally manipulable anonymous social choice functions have strictly more manipulable profiles than one has to admit when demanding only nondictatoriality. This is in contrast to the three alternative case where we have, for $m=3$,

$$
n(m-2)\left(\frac{m !}{3}-1\right)=n<2 n-1=(n-1)\left(\frac{m !}{2}-1\right)+1,
$$


so the anonymous social choice functions outperform the almost dictatorial social choice functions in terms of manipulability. We conclude this section with some proportions of manipulable profiles of social choice functions to give an impression in relative terms of the manipulability that has to be admitted. The following table contains the proportion of profiles that are manipulable for the social choice functions characterized in Theorem 12, the almost dictatorial social choice functions, Plurality and Borda rule. The numbers for the last two are taken from Aleskerov (1999), where tie-breaking according to a fixed order of alternatives is used to decide on ties. We restrict the tables to three and four alternatives. Loosely speaking, these tables indicate that there is still a lot to discover in the space between classical social choice functions and social choice functions obtained by minimizing manipulability. To avoid wrong impressions we note that Slinko (2002) has shown that the proportions of manipulable profiles of Plurality and Borda rule go to zero at a speed of at least $O\left(\frac{1}{\sqrt[2]{n}}\right)$.

\begin{tabular}{|c|c|c|c|c|}
\hline$(m, n)$ & Almost dictatorial & Theorem 12 & Plurality & Borda \\
\hline$(3,2)$ & $8,333333 \%$ & $5,555556 \%$ & $11,11 \%$ & $38,89 \%$ \\
\hline$(3,3)$ & $2,314815 \%$ & $1,388889 \%$ & $16,67 \%$ & $23,61 \%$ \\
\hline$(3,4)$ & $0,540123 \%$ & $0,308642 \%$ & $18,52 \%$ & $31,02 \%$ \\
\hline$(3,5)$ & $0,115741 \%$ & $0,064300 \%$ & $23,15 \%$ & $28,55 \%$ \\
\hline$(3,6)$ & $0,023577 \%$ & $0,012860 \%$ & $23,93 \%$ & $27,82 \%$ \\
\hline$(3,7)$ & $0,004644 \%$ & $0,002501 \%$ & $25,73 \%$ & $27,05 \%$ \\
\hline$(3,8)$ & $0,000893 \%$ & $0,000476 \%$ & $27,39 \%$ & $25,99 \%$ \\
\hline$(3,9)$ & $0,000169 \%$ & $0,000089 \%$ & $27,44 \%$ & $24,98 \%$ \\
\hline$(3,10)$ & $0,000031 \%$ & $0,000017 \%$ & $28,55 \%$ & $24,06 \%$ \\
\hline \hline$(m, n)$ & Almost dictatorial & Theorem 12 & Plurality & Borda \\
\hline$(4,2)$ & $0,173611111111 \%$ & $4,861111111111 \%$ & $20,83 \%$ & $60,42 \%$ \\
\hline$(4,3)$ & $0,014467592593 \%$ & $0,303819444444 \%$ & $29,43 \%$ & $51,22 \%$ \\
\hline$(4,4)$ & $0,000904224537 \%$ & $0,016878858025 \%$ & $32,47 \%$ & $50,02 \%$ \\
\hline$(4,5)$ & $0,000050234697 \%$ & $0,000879107189 \%$ & $37,38 \%$ & $50,44 \%$ \\
\hline$(4,6)$ & $0,000002616390 \%$ & $0,000043955359 \%$ & $38,91 \%$ & $47,90 \%$ \\
\hline$(4,7)$ & $0,000000130820 \%$ & $0,000002136719 \%$ & $40,55 \%$ & $46,43 \%$ \\
\hline$(4,8)$ & $0,000000006359 \%$ & $0,000000101749 \%$ & $41,33 \%$ & $44,85 \%$ \\
\hline$(4,9)$ & $0,000000000303 \%$ & $0,000000004769 \%$ & $41,99 \%$ & $43,15 \%$ \\
\hline$(4,10)$ & $0,000000000014 \%$ & $0,000000000221 \%$ & $41,95 \%$ & $41,78 \%$ \\
\hline
\end{tabular}




\section{Conclusion}

We show that a Gibbard-Satterthwaite like result holds also on sets of profiles with a certain diversity. This is used to characterize the minimally manipulable anonymous social choice functions for more than three alternatives. It turns out that these generalize strategy-proof two alternative imputation status-quo voting, and the minimally manipulable three alternative nondictatorial social choice functions. However, contrary to the three alternative case they are not less manipulable than the almost dictatorial social choice functions. So, they do not constitute the set of minimally manipulable nondictatorial social choice functions. Moreover, the way in which they achieve minimal manipulability is at the expense of treating alternatives unequally. This suggests that a property ensuring a more equal treatment of alternatives should be added. The natural candidate is neutrality, but unfortunately anonymity and neutrality exclude each other whenever the number of alternatives can be written as a sum of non-trivial divisors of the number of agents, see Moulin (1983, p. 25). Nevertheless, this will be an issue for further research, either demanding weaker axioms than neutrality to ensure a more equal treatment of alternatives, or weakening anonymity and demanding neutrality. Also other principles of voting could be added as properties, such as respecting unanimity, Pareto-optimality, absolute plurality, plurality or Condorcet-winners.

\section{References}

[1] Aleskerov F, Kurbanov, E (1999) Degree of manipulability of social choice procedures. In: Proceedings of the Third International Meeting of the Society for the Advancement of Economic Theory. Springer, Heidelberg and New York.

[2] Arrow, K (1963) Social choice and individual values. John Wiley, New York

[3] Dasgupta P, Hammond P, Maskin E (1979) The implementation of social choice rules: Some general results on incentive compatibility. Review of Economic Studies 46: 185-216

[4] Fristrup P, Keiding H (1998) Minimal manipulability and interjacency for two-person social choice functions. Social Choice and Welfare 15: $455-467$ 
[5] Gibbard A (1973) Manipulation of voting schemes: a general result. Econometrica 41: 587-602

[6] Kelly JS (1988) Minimal manipulability and local strategy-proofness. Social Choice and Welfare 5: 81-85

[7] Moulin, H (1983) The strategy of social choice. Advanced Textbooks in Economics 18. North-Holland, Amsterdam, New York and Oxford.

[8] Maus, S, Peters, H, Storcken, A (2004) Minimal manipulability: Unanimity and Nondictatorship

[9] Satterthwaite M (1975) Strategy-proofness and Arrow's conditions: existence and correspondence theorems for voting procedures and social welfare functions. J Econ Theory 10: 187-217.

[10] Slinko, A (2002) On asymptotic strategy-proofness of classical social choice rules. Theory and Decision 52: 389-98 